\title{
Evidence Regarding Rapid Initiation of Antiretroviral Therapy in Patients Living with HIV
}

\author{
Sarah M. Michienzi ${ }^{1}$ (D) Mario Barrios ${ }^{2} \cdot$ Melissa E. Badowski $^{1}$ (D) \\ Accepted: 23 February 2021 / Published online: 2 April 2021 \\ (C) The Author(s), under exclusive licence to Springer Science+Business Media, LLC, part of Springer Nature 2021
}

\begin{abstract}
Purpose of Review Rapid initiation of antiretroviral therapy (ART) is increasingly more common among clinics serving people living with human immunodeficiency virus (PLWH). It is recommended by major guidelines and is especially important in achieving the Getting to Zero (GTZ) goals by 2030. Patients should be offered the option to initiate ART as soon as possible, preferably at time of HIV diagnosis, with the goal of reducing transmission, morbidity, and mortality.

Recent Findings Three published randomized controlled trials, and several other observational, prospective, and retrospective studies, demonstrated superior rates of viral suppression (VS) with initiation of rapid ART compared to standard of care. Improved time to VS and retention in care were also observed. Based on the regimens studied, a tenofovir backbone combined with an integrase strand transfer inhibitor or protease inhibitor is recommended for rapid start initiation. Since ART is started earlier compared with standard of care, there is opportunity to achieve VS at a much faster rate, especially in the setting of starting on the day of diagnosis. What requires further evaluation is whether or not VS is sustained over time with quicker linkage and initiation of HIV care.

Summary Initiating rapid ART in newly diagnosed PLWH provides a promising approach to achieving GTZ. When offered rapid ART, virologic suppression is improved compared to standard of care, which may reduce transmission and, ultimately, new HIV infections.
\end{abstract}

Keywords $\mathrm{HIV} \cdot \mathrm{ART} \cdot$ Antiretroviral $\cdot$ Rapid start $\cdot$ Immediate start

\section{Introduction}

The human immunodeficiency virus (HIV) epidemic has largely improved since the introduction of combination

This article is part of the Topical Collection on Antimicrobial Development and Drug Resistance

Melissa E. Badowski

badowski@uic.edu

Sarah M. Michienzi

msarah@uic.edu

Mario Barrios

mbarri6@uic.edu

1 Department of Pharmacy Practice, Section of Infectious Diseases Pharmacotherapy, University of Illinois at Chicago, College of Pharmacy, Chicago, IL, USA

2 University of Illinois at Chicago, College of Pharmacy, Chicago, IL, USA antiretroviral therapy (cART). Based on data derived from the START and TEMPRANO trials, treatment guidelines expanded recommendations to initiate ART in all people living with HIV (PLWH) regardless of CD4 count [1, 2]. Furthermore, the evolution of safer and more effective cART has helped patients improve immunologic function, achieve virologic suppression, reduce morbidity and mortality, and improve overall quality of life [3•].

Despite initiatives such as Treatment as Prevention (TasP), Getting to Zero (GTZ), Undetectable=Untransmittable $(\mathrm{U}=\mathrm{U})$, Ending the HIV Epidemic (EHE), and Preexposure Prophylaxis (PrEP), new infections of HIV are still occurring. This is especially true among certain risk groups and geographic areas, such as young Black men who have sex with men (MSM) and the Southern United States [4]. Initiation of ART as soon as possible, ideally at time of diagnosis, may assist in reducing HIV incidence. Previous first-line ART regimens with non-nucleoside reverse transcriptase inhibitors (NNRTIs) were marred by high rates of virologic failure and 
transmitted drug resistance secondary to lower genetic barriers to resistance compared to protease inhibitor (PI)-based therapy or second-generation integrase strand transfer inhibitor (INSTI)-based therapy (i.e., bictegravir and dolutegravir).

Although current evidence recommends obtaining a genotype prior to initiation of ART, transmitted drug resistance to regimens used in rapid (within days or weeks of diagnosis, but ideally within 2 weeks) or immediate start (day of diagnosis) scenarios are rarely associated with mutations (Fig. 1) [5]. Therefore, a genotype can still be obtained the same-day ART is initiated and ART can be modified based on results, if needed. While this practice has the potential to reduce the time to achievement of virologic suppression, and subsequently, the time to which newly diagnosed patients with HIV cannot transmit the virus sexually, various logistical barriers can prevent it from occurring (i.e., lack of active insurance, affordability, etc.). The goal of this manuscript is to review available evidence on rapid or immediate initiation of ART in newly diagnosed PLWH.

\section{Methods}

A literature search for clinical trials in the English language of adults living with HIV and associated clinical outcomes (virologic suppression and/or retention in care), was conducted utilizing Medline, Google Scholar, Embase, conference proceedings, and bibliographies within 5 years of July 28, 2020, using the following terms: HIV, ART, rapid start, rapid initiation, immediate start, and same-day initiation. Ongoing clinical trials were obtained from the US National Library of Medicine through the database of ClinicalTrials.gov using the same search terms mentioned above.

\section{Results}

Of 113 clinical trials and abstracts reviewed, 7 studies were included in this review $[6 \bullet \bullet, 7-9,10 \bullet, 11 \bullet \bullet, 12 \bullet \bullet$.

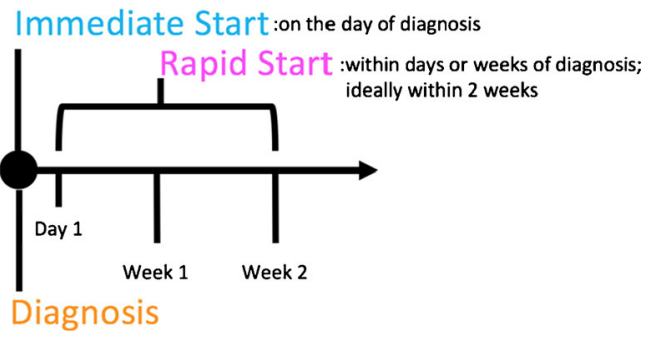

Fig. 1 Immediate vs. rapid start

\section{Published Studies}

\section{RaplT Trial}

The first study to report on rapid start was the Rapid Initiation of Treatment (RapIT) trial, which evaluated standard management of HIV compared to rapid ART initiation (Table 1) [6・•]. Enrolled patients presented for an HIV test to determine status or, if known to have HIV, presented for CD4 count to determine treatment eligibility. Any patient that was previously eligible for ART based on CD4 count or had previously taken ART was excluded from the study. Virologic suppression at 10 months was achieved in significantly more patients in the rapid arm vs. the standard of care arm. For patients in the rapid arm that initiated ART after 90 days of study enrollment, rate of virologic suppression was dramatically reduced. Ultimately, this study revealed that patients benefited from initiating ART within 90 days compared to standard care.

\section{Rapid HIV Viral Load Suppression}

A subsequent study evaluated the impact of early initiation ART and regimen type vs. time to viral suppression (Table 1) [7]. By week 12 post-initiation of rapid ART, significantly more patients achieved virologic suppression, which was maintained through week 48. Patients receiving INSTI-based therapy achieved virologic suppression in a significantly shorter time than those receiving PI-based therapy (12 vs. 34 weeks; $p=0.022$ ). This study demonstrated that patients who initiated same-day ART with INSTI-based therapy were more likely to achieve virologic suppression and at a faster rate.

\section{Same-Day ART vs. Standard of Care}

A third trial evaluated standard ART initiation vs. same-day HIV testing and ART initiation with an NNRTI-based single tablet regimen, unless dose adjustment was required for renal function (Table 1) [8]. Significantly more patients in the sameday ART group remained in care and achieved virologic suppression compared to the standard of care group at 12 months after testing positive for HIV. This study demonstrated that PLWH with early WHO stage disease and a CD4 count $\leq 500$ cells $/ \mathrm{mm}^{3}$ had improved retention in care and virologic suppression when started on same-day ART compared to standard care of treatment. The development of resistance was not reported in this study.

\section{CASCADE Trial}

The CASCADE study offered same-day, home-based ART initiation after new HIV diagnosis (Table 1) [9]. Rates of linkage to care within 3 months and virologic suppression at 


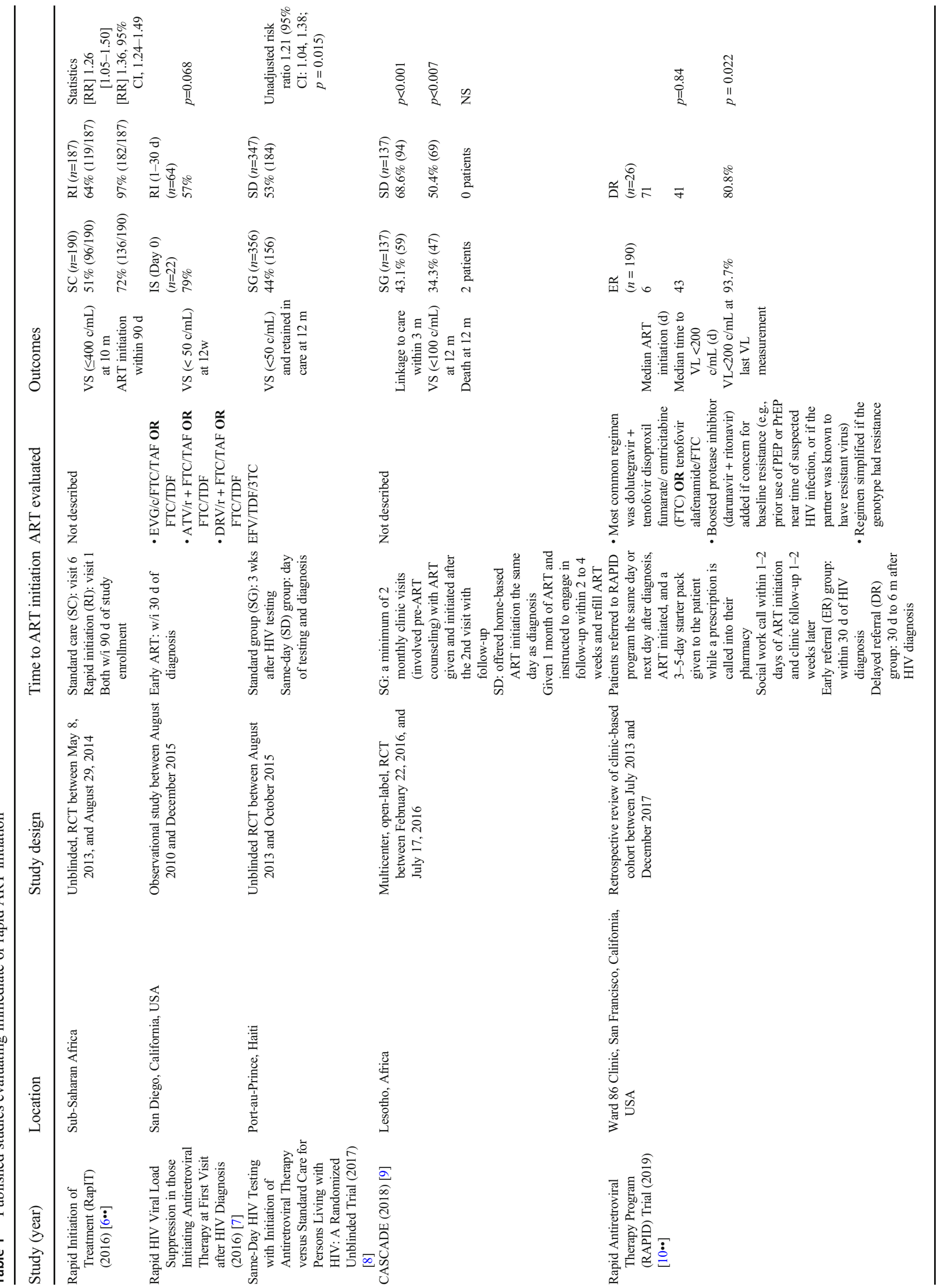




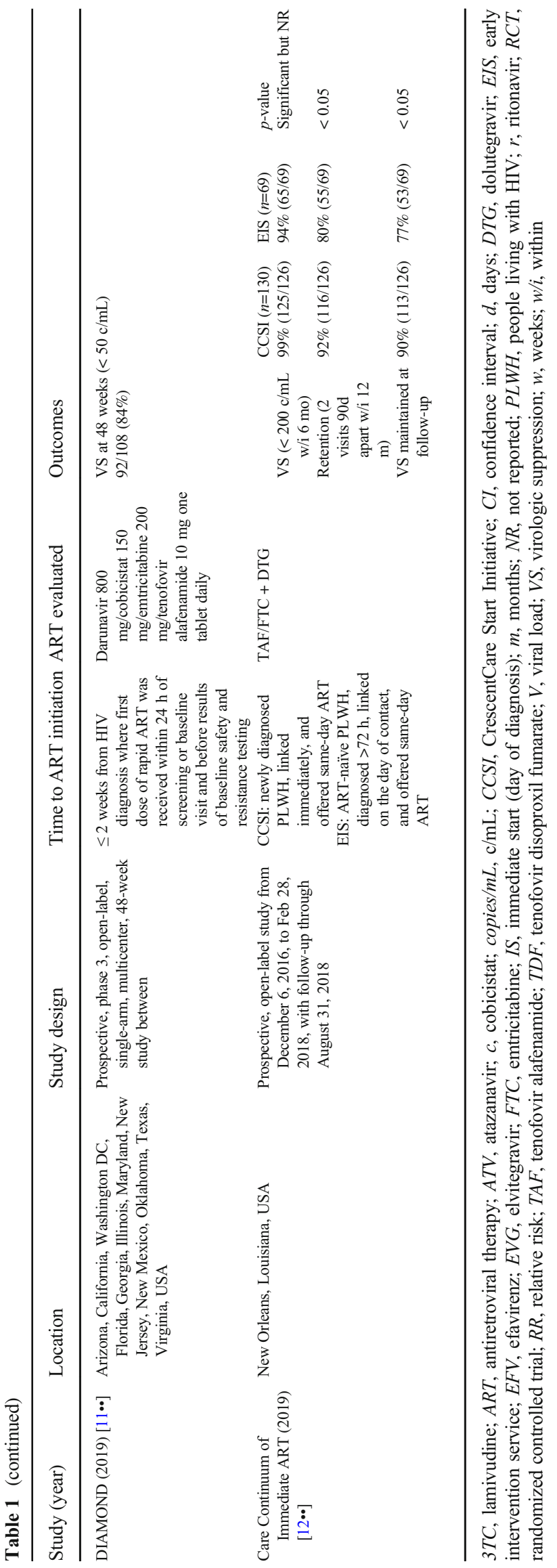

12 months were significantly higher in the same-day ART group compared to the usual care group. Overall, same-day, home-based ART initiation displayed beneficial results in newly diagnosed PLWH.

\section{RAPID Trial}

The Rapid Antiretroviral Therapy Program (RAPID) trial examined immediate ART initiation after a confirmed HIV diagnosis within a high-risk population (Table 1) [10••]. Of note, $51 \%$ had a substance use disorder, $48 \%$ had a major mental health disorder, and $31 \%$ were homeless. The overall median time from HIV diagnosis to ART start was approximately 7 days and the time from the first RAPID visit to ART initiation was 0 days for all patients regardless of early or delayed referral. Within 1 year after initiating ART, 95.8\% of the entire population achieved virologic suppression. Patients in the early referral group achieved virologic suppression at a significantly higher rate and significantly faster than the delayed group. Factors that lead to a poorer virologic response within the delayed referral group were higher rates of mental health disorders, substance use, and homelessness. This model proved that rapid initiation can be accomplished even when barriers are present.

\section{DIAMOND Trial}

The DIAMOND trial was the first trial to evaluate rapid start with darunavir/cobicistat/emtricitabine/tenofovir alafenamide (D/C/F/TAF) (Table 1) [11••]. Of note, the majority of study participants (75\%) were young MSM. Virologic suppression was achieved in the majority of the population at week 48 and the majority were satisfied with their treatment and would recommend it to other PLWH (97\% and 98\%, respectively). There were no DRV or TAF resistance-associated mutations (RAMs) observed. Two participants had M184V/I and five had a primary PI RAM, but retained DRV sensitivity. In addition, five participants had secondary transmitted INSTI resistance at baseline with T97. Ultimately, the DIAMOND study demonstrated that $\mathrm{D} / \mathrm{C} / \mathrm{F} / \mathrm{TAF}$ was an appropriate and safe regimen for rapid ART initiation.

\section{Care Continuum of Immediate ART}

A health center in New Orleans evaluated two rapid-start interventions over the same time period using TAF/FTC + DTG in a Southern community clinic. (Table 1) [12••]. The CrescentCare Start Initiative (CCSI) offered immediate linkage and ART start to patients diagnosed less than $72 \mathrm{~h}$ prior while Early Intervention Services (EIS) offered linkage and ART start to patients diagnosed more than $72 \mathrm{~h}$ prior. Deviations from TAF/FTC + DTG were rare with only five patients receiving a different regimen. No patients switched 
regimens due to the development of renal or hepatic abnormalities. Patients in the CCSI group had significantly better retention in care and VS compared to patients in the EIS group $(p<0.05)$. Additionally, median CD4 count was significantly higher in the CCSI group $(p<0.05)$ while mental health diagnoses were significantly higher in the EIS group $(p<0.05)$. All patients in both groups with transmitted resistance achieved VS. Overall, NNRTI mutations were most common and 4 patients developed M184I/V RAM. These models demonstrated the benefit of ART initiation on the day of linkage, or ideally, on the day of diagnosis. Additionally, they support rapid-start implementation in community-based clinics.

\section{Conference Abstracts}

Although rapid start models are being explored globally, many studies presented at conferences were merely descriptive in nature and not associated with outcome data (i.e., VS, time to VS, and linkage to care). Of the rapid ART start models presented in 2020, most were associated with significant reductions in time to VS, linkage to care, and increases in the number of patients achieving VS when compared to previous standards of care (Table 2) [13-18]. What remains to be seen is whether continued linkage to care and VS is sustained, especially during the time of the coronavirus pandemic.

\section{Ongoing Trials}

There are many ongoing trials to evaluate rapid or immediate ART start in a variety of populations in the USA and abroad. While a full appraisal of these studies falls outside of the scope of this review, it is worth highlighting a small selection. One such trial is the Rapid HIV Treatment Initiation, Access and Engagement in Care (RHAE) study [19]. This pilot study seeks to investigate uptake and acceptability of rapid initiation of TAF/FTC + DTG in newly and previously diagnosed but out of care PLWH in Baltimore. The goal is to generate a model that can be generalized and implemented in similar cities. Another study in Portugal will compare VS between a prospective cohort of newly diagnosed PLWH enrolled in a test and treat model compared to a historical cohort [20]. Specific ART regimens will be selected according to current local standards and guidelines. The Prospective Pilot Study of the Efficacy, Safety and Tolerability of Bictegravir-based HIV ART Same-Day Treatment Evaluations (B-HASTE) occurring in Colorado and Nebraska will compare VS between same-day start of BIC/TAF/FTC when compared to standard initiation of ART at the discretion of the provider [21]. Both groups will be given a new diagnosis package and social work referral. This final example is a multi-center study in the USA that will evaluate VS through a rapid test and treat model with the two-drug regimen of DTG/3TC [22]. To date, only threedrug regimens have been evaluated and recommended for rapid start. Results of these ongoing studies have the potential to confirm current knowledge and offer insight into expanded patient populations and/or regimens for rapid-start of ART.

\section{Guidelines}

The four major organizations that provide guidelines for the management of HIV, the Department of Health and Human Services (DHHS) [3•], European AIDS Clinical Society (EACS) [23•], International Antiviral Society (IAS) [24•], and World Health Organization (WHO) [25•], are in agreement and support recommendations for rapid start of ART. Specific recommendations, along with strength of recommendation and the quality of evidence scale when available, are provided in Table 3. While wording varies slightly between the guidelines, each recommends rapid ART initiation within 7-14 days of diagnosis with immediate, same-day start, when possible.

\section{Discussion}

While many studies describe their models, very few provide outcome data such as virologic suppression, which is necessary to classify risk-reduction. Clinical evaluations and guidelines support rapid initiation of ART at or within 14 days of HIV diagnosis $[3,23 \bullet, 24 \bullet, 25 \bullet$. Various trials demonstrated a significant decrease in time to VS when same-day ART was initiated $[8,9]$. Other trials also demonstrated that when ART was initiated within 7 to 14 days, patients were more likely to achieve VS when compared to patients starting ART after 14 days $[10 \bullet \bullet, 11 \bullet \cdot]$.

One of the major differences within the literature and guidelines is the lack of universal terminology between rapid and immediate start (Fig. 1). Rapid initiation is the general terminology for initiating ART within 14 days of HIV diagnosis whereas immediate initiation is defined as initiating ART on the same day of diagnosis. Clinical trial data demonstrated an increased likelihood and quicker achievement of VS in patients receiving INSTI-based therapy (TAF/FTC or TDF/ FTC combined with EVG/COBI or DTG) when compared to PI-based therapy (TAF/FTC or TDF/FTC combined with ritonavir-boosted atazanavir or darunavir) [7, 12••]. Although the use of efavirenz was evaluated as a potential agent in rapid ART initiation, it is not an ideal agent due to its high rates of transmitted drug resistance and development of RAMs [26, 27]. It is important to note that many models that were evaluated lacked the specifics of ART used which is essential if starting in the absence of a genotype. In addition, abacavir should never be used in a rapid initiation protocol due to the risk for a life-threatening hypersensitivity reaction if positive for HLA-B*5701. Agents used in the management of heavily treatment experienced patients (i.e., enfuvirtide, 


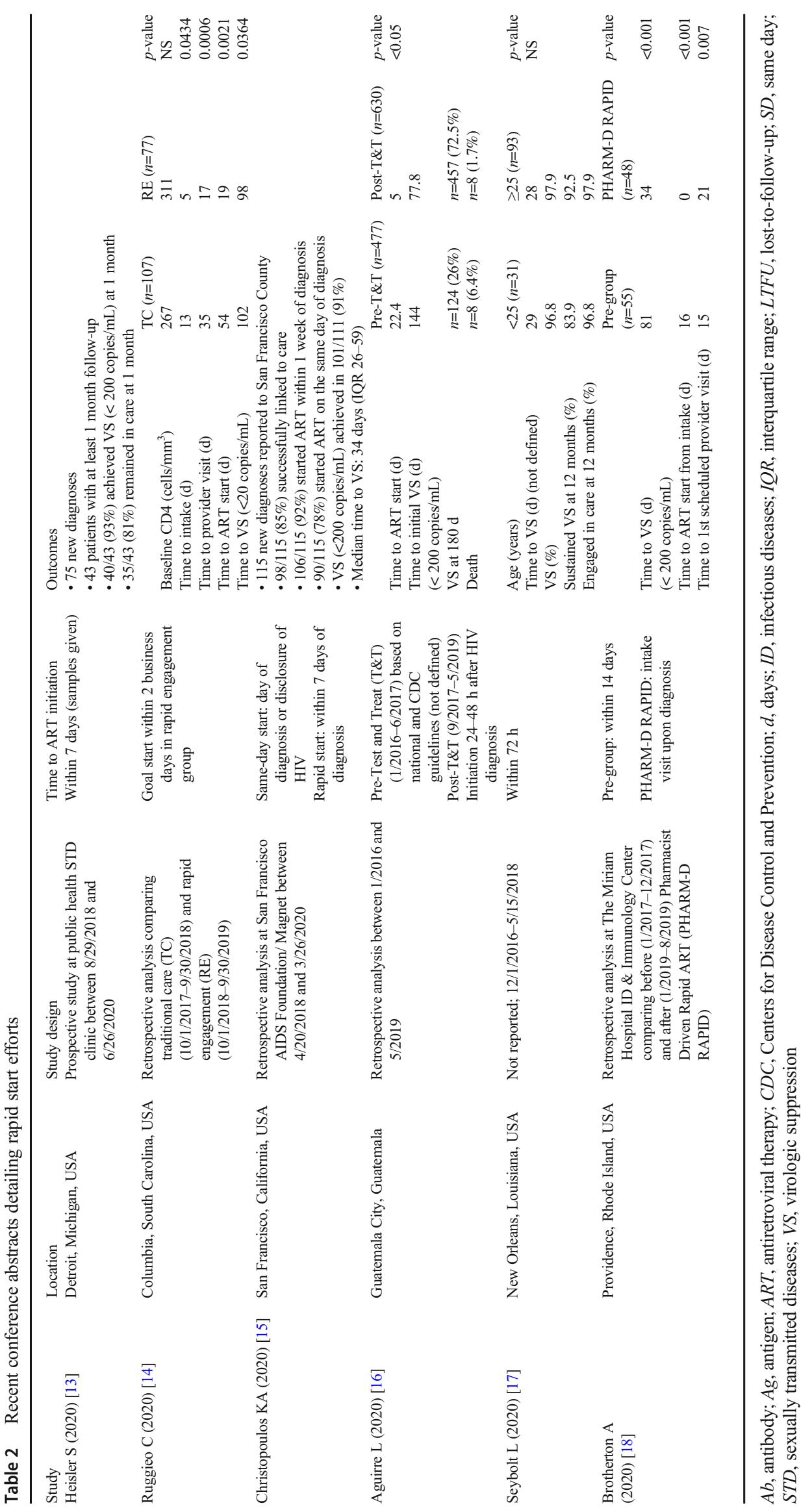


Table 3 HIV guideline recommendations for rapid start of antiretrovirals

\begin{tabular}{|c|c|}
\hline Guideline organization & Recommendation \\
\hline $\begin{array}{l}\text { DHHS [3•] } \\
\text { (December 2019) }\end{array}$ & $\begin{array}{l}\text { - ART is recommended for all PLWH } \\
\text { - Initiate ART immediately (same day), or as soon as possible (within days or weeks; } \\
\text { rapid) } \\
\text { - Strong recommendations, data from RCT and well-designed non-RCTs or } \\
\text { observational cohorts with long-term outcomes, respectively }\end{array}$ \\
\hline $\begin{array}{l}\text { EACS [23•] } \\
\text { (Version 10.0, } \\
\quad \text { November 2019) }\end{array}$ & $\begin{array}{l}\text { - Starting ART is recommended regardless of CD4 } \\
\text { - Assess "stage of readiness to start" using the tool provided" } \\
\text { - Consider immediate (same day) start of ART, especially: } \\
\text { - Primary HIV infection, especially in the case of meningoencephalitis } \\
\text { o Patient is interested } \\
\text { o Loss-to-follow-up is more likely if ART is delayed }\end{array}$ \\
\hline $\begin{array}{l}\text { IAS [24•] } \\
\text { (October 2020) }\end{array}$ & $\begin{array}{l}\text { - ART should be initiated as soon as possible after diagnosis, including immediately, } \\
\text { unless not ready to commit to ART } \\
\text { - For most OIs, start ART as soon as possible but within the first } 2 \text { weeks } \\
\text { - Strong support for the recommendation, evidence form } \geq 3 \text { peer-reviewed RCT }\end{array}$ \\
\hline $\begin{array}{l}\text { WHO [25•] } \\
\text { (July 2017) }\end{array}$ & $\begin{array}{l}\text { - Rapid ART initiation within } 7 \text { days of confirmed HIV diagnosis is recommended } \\
\text { for all PLWH } \\
\text { - Same-day ART initiation recommended for PLWH who are ready to start } \\
\text { - Strong recommendations, high-quality evidence for adults and adolescents }\end{array}$ \\
\hline
\end{tabular}

$A R T$, antiretroviral therapy; DHHS, Department of Health and Human Services; EACS, European AIDS Clinical Society; HIV, human immunodeficiency virus; IAS, International Antiretroviral Society; OIs, opportunistic infections; $P L W H$, people living with HIV; RCT, randomized controlled trial; WHO, World Health Organization

https://eacs.sanfordguide.com/art/readiness-to-start-maintain-art fostemsavir, ibalizumab-uiyk, and maraviroc) should be preserved for later consideration.

Rapid initiation of ART is not a one size fits all approach. Although it is ideal to initiate ART as soon as possible, there are certain situations which warrant deferred treatment such as patients with untreated opportunistic infections (OIs) where ART initiation should be delayed for a short period to reduce the development of immune reconstitution inflammatory syndrome (IRIS) (i.e., cryptococcal meningitis, tuberculosis meningitis, cytomegalovirus retinitis, or other central nervous system OIs with inflammation) [28]. Initiation of ART before or at the same time as tuberculosis treatment increases the risk of IRIS, especially if patients have a low CD4 cell count [29].

Likewise, patient preference and motivation serves as another indicator on whether rapid ART should be initiated in a newly diagnosed PLWH [30]. Patients that may want to defer treatment include those unwilling or unready to commit to lifelong ART. These patients should still be engaged in follow-up as many choose to initiate ART at subsequent visits. A study performed in Rwanda found patients initiating treatment on the same day as diagnosis were more likely to be lost to follow-up compared to those initiating ART later [31]. Additionally, one study evaluating patient interviews found PLWH chose to delay ART initiation due to perceived cost barriers, wanting additional medical consultation, or time to consider ART [32]. Other patients reported reasons for wanting to start ART immediately included fear of not being treated, personal health, and influence by other people and clinical staff.

Patients starting immediate ART should also meet certain criteria conditions. These conditions include patients with a confirmed new diagnosis of HIV, suspected acute HIV, positive rapid HIV antibody test and awaiting a confirmed diagnosis, and chronically infected PLWH who are naive to treatment or returning to care after lost-to-follow-up based on their treatment history [28]. With these patients, a standard protocol within the first immediate ART clinic visit should consist of patient education, emotional support, and baseline laboratory results including HIV-1 viral load; CD4 cell count; HIV-1 genotype; HLA-B*5701 allele test; hepatitis A, B, and C serologies; sexually transmitted infections; fasting blood glucose or hemoglobin A1C; and a fasting lipid panel [28].

A noticeably underrepresented group within these studies include women as most of these rapid interventions occur in MSM population. Most successful rapid start programs verify insurance and prescription drug coverage prior to the patient picking up medication. This is extremely important as the patient may not understand the need for a prior authorization, lack of meeting a deductible, or even loss of insurance. Many patients may find it difficult or embarrassing if they are turned away at the pharmacy due to the inability to pay a co-payment or deductible or understand the issues that is occurring with 
their prescription. This is yet another barrier to ART which can easily be avoided with appropriate communication and follow-up.

\section{Conclusion}

In achieving GTZ, not only must rapid start practices be universal and available for all, another essential area to explore is initiating rapid re-entry into care for patients lost to follow-up. While this scenario would be more difficult based on unknown treatment history and/or genotype/phenotype data, it would accelerate re-engagement into HIV care, provide VS in those interested in therapy, and reduce HIV transmission.

Code Availability Not applicable.

Author Contribution All authors contributed to the study conception and design. Material preparation, data collection, and analysis were performed by Sarah Michienzi, Mario Barrios, and Melissa Badowski. The first draft of the manuscript was written by Sarah Michienzi, Mario Barrios, and Melissa Badowski and all authors commented on previous versions of the manuscript. All authors read and approved the final manuscript.

Data Availability Not applicable.

\section{Declarations}

Ethics Approval Not applicable.

Consent to Participate Not applicable.

Consent for Publication The authors of this manuscript consent to publication of this original work.

Conflict of Interest Dr. Michienzi has received grant funding from Merck.

Human and Animal Rights and Informed Consent This article does not contain any studies with human or animal subjects performed by any of the authors.

\section{References}

Papers of particular interest, published recently, have been highlighted as:

- Of importance

• Of major importance

1. INSIGHT START Study Group, Lundgren JD, Babiker AG, et al. Initiation of antiretroviral therapy in early asymptomatic HIV infection. N. Engl. J. Med. 2015;373(9):795-807 Available at: http:// www.ncbi.nlm.nih.gov/pubmed/26192873.
2. TEMPRANO ANRS Study Group, Danel C, Moh R, et al. A trial of early antiretrovirals and isoniazid preventive therapy in Africa. N. Engl. J. Med. 2015;373(9):808-22 Available at: http://www.ncbi. nlm.nih.gov/pubmed/26193126.

3. Panel on Antiretroviral Guidelines for Adults and Adolescents. Guidelines for the use of antiretroviral agents in adults and adolescents with HIV. Department of Health and Human Services. Available at http://aidsinfo.nih.gov/contentfiles/lvguidelines/ AdultandAdolescentGL.pdf. Accessed 22 Jan 2021. United States based guidelines that provides evidence for the management of HIV. Provides recommendations for antiretroviral therapy as it relates to dosing, side effects, drug interactions, and monitoring parameters.

4. Centers for Disease Control and Prevention. Statistics overview. Available at https://www.cdc.gov/hiv/statistics/overview/index. html. Accessed 25 Aug 2020.

5. Rhee SY, Clutter D, Fessed WJ, et al. Trends in the molecular epidemiology and genetic mechanisms of transmitted human immunodeficiency virus type 1 drug resistance in a large US clinic population. Clin. Infect. Dis. 2019;68(2):213-21. https://doi.org/ 10.1093/cid/ciy453.

6.• Rosen S, Maskew M, Fox MP, Nyoni C, Mongwenyana C, Malete $\mathrm{G}$, et al. Initiating antiretroviral therapy for HIV at a patient's first clinic visit: the RapIT randomized controlled trial. PLoS Med. 2016;13(5):e1002015. https://doi.org/10.1371/journal.pmed. 1002015. This study performed in South Africa evaluated the role of initiating antiretroviral therapy at a patient's initial clinic visit. Based on this practice, the study demonstrated increased uptake of antiretroviral therapy by $36 \%$ and viral suppression by $26 \%$.

7. Hoenigle M, Chaillon A, Moore DJ, et al. Rapid HIV viral load suppression in those initiating antiretroviral therapy at first visit after HIV diagnosis. Sci. Rep. 2016;6:3297. https://doi.org/10. 1038/srep32947.

8. Koenig SP, Dorvil N, Devieux JG, et al. Same-day HIV testing with initiation of antiretroviral therapy versus standard care for persons living with HIV: a randomized unblinded trial. PLoS Med. 2017;14(7):e1002357. https://doi.org/10.1371/journal.pmed. 1002357.

9. Labhardt ND, Ringera I, Lejone TI, Klimkait T, Muhairwe J, Amstutz A, et al. Effect of offering same-day ART vs usual health facility referral during home-based HIV testing on linkage to care and viral suppression among adults with HIV in Lesotho: the CASCADE randomized clinical trial. JAMA. 2018;319(11): 1103-12. https://doi.org/10.1001/jama.2018.1818.

$10 . \bullet$ Coffey S, Bacchetti P, Sachdev D, Bacon O, Jones D, OspinaNorvell C, et al. RAPID antiretroviral therapy: high virologic suppression rates with immediate antiretroviral therapy initiation in a vulnerable urban clinic population. AIDS. 2019;33:825-32. https:// doi.org/10.1097/QAD.0000000000002124. This retrospective analysis evaluated 225 patients who were referred for rapid antiretroviral therapy. 216 patients started immediate antiretroviral therapy. Although this clinic had high rates of mental illness, substance use, and unstable housing, more than $90 \%$ of patients achieved viral suppression over a median of 1 year.

11.• Huhn GD, Crofoot G, Ramgopal M, Gathe J Jr, Bolan R, Luo D, et al. Darunavir/cobicistat/emtricitabine/tenofovir alafenamide in a rapid initiation model of care for HIV-1 infection: primary analysis of the DIAMOND study. Clin. Infect. Dis. 2019;71:3110-7. https:// doi.org/10.1093/cid/ciz1213. This was the first study to evaluate a specific antiretroviral regimen consisting of darunavir/ cobicistat/emtricitabine/tenofovir alafenamide for rapid initiation of antiretroviral therapy. This prospective, Phase 3 clinical trial evaluated the efficacy and safety of this single tablet regimen. At week 48, of the 97 participants who 
completed the study, 92 achieved virologic suppression and few discontinued therapy.

12.• Halperin J, Conner K, Butler I, et al. A care continuum of immediate ART for newly diagnosed patients and patients presenting later to care at a federally qualified health center in New Orleans. Open Forum Infect Dis. 2019;6(4):ofz161. https://doi.org/10.1093/ofid/ ofz161. This is the first manuscript describing the continuum of care for a rapid-start model in the United States. Nearly all patients accepted treatment during their first visit in both cohorts. Viral suppression was achieved in $99.2 \%$ of patients and transmitted drug resistance did not impact viral suppression.

13. Heisler S, Cohn J, Lukomski D, Tuinier K. Ending the HIV epidemic: rapid ART start at the Detroit STD Clinic. Poster presented at: IAS 2020; July 6 - 10, 2020; Virtual.

14. Ruggiero C, Patel P, Derrick C, Ahuja D, Weissman S, Milgrom A. Impact of rapid HIV engagement program in a Southern United States clinic. Poster presented at: IAS 2020; July 6 - 10, 2020; Virtual.

15. Christopoulos KA, Crouch PC, Pilcher C, LeTrourneau N, Defechereux P, Schmitto C, et al. High rates of nurse-led rapid ART start and subsequent linkage to care/viral suppression at a large community-based HIV testing and prevention site in San Francisco, California, USA. Poster presented at: IAS 2020; July 6 $-10,2020$; Virtual.

16. Aguirre L, Brown C, Perez JC, Ortiz B, Mercado D, Arathoon E, Samayoa B. Impact of rapid ART initiation in recently diagnosed patients in an HIV reference public clinic of Guatemala. Poster presented at: IAS 2020; July 6 - 10, 2020; Virtual.

17. Seybolt L, Conner K, Butler I, Van Sickels N, Halperin J. Rapid start leads to sustained viral suppression in young people in the South. Poster presented at: CROI 2020; March 8 - 11, 2020; Virtual.

18. Brotherton A, Shah RB, Garland J, McCarthy ML, Gillani FS, Sanchez MC. Pharmacist-driven rapid ART reduces time to virologic suppression in Rhode Island. Poster presented at: CROI 2020; March 8-11, 2020; Virtual.

19. ClinicalTrials.gov [Internet]. Bethesda (MD): National Library of Medicine (US). 2018 May 1 - . Identifier NCT03512964, Rapid HIV Treatment Initiation, Access and Engagement in Care; 201929 [cited 2020 October 23]. Available from: https://www. clinicaltrials.gov/ct2/show/NCT03512964?term=HIV+Rapid+ initiation\&draw $=2 \&$ rank=5. Accessed 22 Jan 2021.

20. ClinicalTrials.gov [Internet]. Bethesda (MD): National Library of Medicine (US). 1 November 2019 - . Identifier NCT04147325, A study to evaluate virologic response in participants newly diagnosed with HIV-1; 2020 [cited 2020 October 23]. Available from: https://www.clinicaltrials.gov/ct2/show/NCT04147325?term= HIV+Rapid+initiation\&draw=2\&rank=4. Accessed 22 Jan 2021.

21. ClinicalTrials.gov [Internet]. Bethesda (MD): National Library of Medicine (US). 30 January 2020 -. Identifier NCT04249037, Rapid start vs. standard start antiretroviral therapy (ART) in HIV (BHASTE); 2022 March 1 [cited 2020 November 24]. Available from: https://clinicaltrials.gov/ct2/show/study/NCT04249037? term=b-haste\&draw=2\&rank=1. Accessed 22 Jan 2021.

22. ClinicalTrials.gov [Internet]. Bethesda (MD): National Library of Medicine (US). 10 May 2019 - . Identifier NCT03945981, Rapid test and treat dolutegravir plus lamivudine study in newly diagnosed human immunodeficiency virus (HIV)-1 infected adults; 27 April 2020 [cited 2020 November 24]. Available from https://
clinicaltrials.gov/ct2/show/study/NCT03945981?term=Rapid+ start\&cond=HIV\&draw=2. Accessed 22 Jan 2021.

23. European AIDS Clinical Society. (2020). Readiness to start/ maintain ART. Available from: https://eacs.sanfordguide.com/art/ readiness-to-start-maintain-art. Accessed 22 Jan 2021. This document provides an overview on important considerations of starting or maintaining a patient on antiretroviral therapy based on recommendations from the European AIDS Clinical Society.

24. Saag MS, Gandhi RT, Hoy JF, Landovitz RJ, Thompson MA, Sax $\mathrm{PE}$, et al. Antiretroviral drugs for treatment and prevention of HIV Infection in Adults: 2020 recommendations of the International Antiviral Society-USA Panel. JAMA. 2020;324(16):1651-69. https://doi.org/10.1001/jama.2020.17025. This practice guideline serves as an update to the 2018 version set forth by the International Antiviral Society-USA. This guideline outlines new evidence and advances in HIV prevention and treatment management.

25. World Health Organization. (2017). Guidelines for managing advanced HIV disease and rapid initiation of antiretroviral therapy. Available from: https://www.who.int/hiv/pub/guidelines/ advanced-HIV-disease/en. Accessed 22 Jan 2021. This guideline provides recommendations on the management of advanced HIV disease as well as the role for rapid initiation of antiretroviral therapy according to the World Health Organization.

26. Snedecor SJ, Khachatryan A, Nedrow K, Chambers R, Li C, Haider $\mathrm{S}$, et al. The prevalence of transmitted resistance to first generation non-nucleoside reverse transcriptase inhibitors and its potential economic impact in HIV infected patients. PLoS One. 2013;8(8): e72784. https://doi.org/10.1371/journal.pone.0072784.

27. Wensing AM, Calvez V, Ceccherini-Silberstein F, et al. 2019 Update of the drug resistance mutations in HIV-1. 2019 Resistance Mutations Update Volume 27 Issue 3 July/August 2019. i-xi.

28. Coffey S, Bacon O. Immediate initiation of antiretroviral therapy in the outpatient clinic. http://hivinsite.ucsf.edu/InSite?page $=$ mdward86-rapid-art. (2020). Accessed 23 Jan 2020.

29. Rosen S, Maskew M, Brenna AT, et al. Improved simplified clinical algorithm for identifying patients eligible for immediate initiation of antiretroviral therapy for HIV (SLATE II): protocol for a randomized evaluation. Trials. 2018;19:548. https://doi.org/10. 1186/s13063-018-2928-5.

30. Christopoulos KA, Erguera XA, VanderZanden L, et al. "Why start later if I can start today?" Patient perspectives on the experience of rapid/same-day linkage and antiretroviral therapy after HIV diagnosis. Poster presented at: IAS 2020; July 6 - 10, 2020; Virtual.

31. Ross J, Murenzi G, Hoover DR, et al. Association between time to ART and loss to care among newly diagnosed PLWH in Rwanda. Poster presented at: CROI 2020; March 8 - 11, 2020; Virtual.

32. Amico KR, Miller J, Schairer C, Gianella S, Little SJ, Hoenigl M. I wanted it as soon as possible: a qualitative exploration of reactions to access to same-day ART start among participants in San Diego's ART-NET project. AIDS Care. 2019;12:1-7. https://doi.org/10. 1080/09540121.2019.1687831.

Publisher's Note Springer Nature remains neutral with regard to jurisdictional claims in published maps and institutional affiliations. 